\title{
Multiplicity in combustion wave behaviour for a model with competing exothermic reactions
}

\author{
$\underline{\text { I. N. Towers }}^{\text {a }}$, H. S. Sidhu ${ }^{\text {a }}$, V. V. Gubernov ${ }^{b}$, A. V. Kolobov ${ }^{\mathrm{b}}$ and A. A. Polezhaev $^{\mathrm{b}}$ \\ ${ }^{a}$ School of Physical, Environmental and Mathematical Sciences, University of New South Wales, Northcott \\ Drive, Canberra ACT 2600 Australia
${ }^{b}$ I. E. Tamm Theory Department, P. N. Lebedev Physical Institute of the Russian Academy of Sciences, 53 \\ Leninsky prospect, Moscow, 119991 Russia \\ Email: i.towers@adfa.edu.au
}

\begin{abstract}
Combustion, while being part of our everyday experience, is a complex phenomenon involving a multitude of reactions and dynamics. There is great value in understanding combustion through mathematical modelling, not only for its own sake, but also because of the applications of the developed model to combustion-based industrial processes or to that perennial environmental threat to populace, property and wildlife — the bushfire.

In this work, we present a mathematical model of a combustive process where the combustion wave propagates through a fuel shaped like a rod, as it may be in self-propagating high temperature synthesis of metals. Said fuel is assumed to have chemical properties such that all reactions occurring during combustion may be lumped together as two different heat producing (exothermic) reactions. The model presented considers the idealised case of there being no heat lost in the reactions (i.e. the adiabatic limit).

The two reactions, say $A$ and $B$, compete for the available fuel. In one limit of the model parameters $A$ will dominate and consume the majority of the fuel. At another extreme reaction $B$ will dominate. In both cases our model reduces to the well-known "one-step" model. The one-step model allows a single wave front solution per system parameter.

At intermediate parameter values no reaction dominates to the complete exclusion of the other. We argue there must exist some parameter value at which reactions $A$ and $B$ are consuming the fuel equally. Either side of this "cross-over" point one reaction begins to dominate. The "cross-over" concept enables the determination of the region in parameter space that allows a multiplicity of solutions. That is, for a given activation energy, there are multiple allowable combustion waves which typically have large differences in propagation speed.

Lastly, the boundary for the transition from unique solutions to multiple solutions is calculated numerically and it is demonstrated that within this multiplicity region the flame front speed is an ' $\mathrm{S}$ '-shaped function of the model parameters.
\end{abstract}

Keywords: Combustion waves, competitive reactions, multiplicity 


\section{INTRODUCTION}

Real-world combustion processes involved a great many chemical reactions occurring sequentially or concurrently. Modelling such a multitude of reactions to understand the combustion process could easily prove overwhelming and would be intractable from an analytic point of view. Insight, however, can be gained by "lumping" reactions together which have the same reaction kinetics. At its simplest, this lumping results in one-step reaction models where it is assumed that the reaction is well described by a single step of fuel and oxidant combining to produce products and heat.

Although simple, one-step models have proved their worth in understanding combustion processes (Zeldovich et al., 1985). Their simplicity means that asymptotic techniques can be brought to bear and the models can be investigated analytically. However, in the majority of combustion processes the chemical reactions proceed according to multiple steps and Westbrook and Dryer (1981) have noted that one-step models may led to incorrect conclusions for many reactions. These issues have lead to the need to develop multi-step models as a means to understand combustion waves.

Useful multi-step models can be formulated by reducing the detailed kinetics of the combustion process through the same lumping concept employed for the one-step models. The models are then formulated around the reaction paths present in the combustion: 'sequential' where the reactions occur one after another (Please et al, 2003); 'parallel' where reactions occur simultaneously but consume different reactants (Ball et al., 1999); and 'competitive' where reactions occur at the same time and consume the same reactants (Gubernov et al., 2012; Sharples et al., 2012).

In earlier works which have considered competitive reaction paths the focus has been on a competition between an exothermic reaction and an endothermic one. In this paper we focus on the investigation of premixed combustion waves in a model with two-step competing exothermic reactions. Combustion waves with twostage competing exothermic reactions $A \rightarrow B$ and $A \rightarrow C$ have been studied experimentally (Martirosyan et al., 1983a,b) and analytically using asymptotic techniques (Berman and Riazantsev, 1975; Clavin et al., 1987). Importantly, Martirosyan et al. (1983a,b) experimentally observed bistability of flame propagation for combustion of Me-C- $\mathrm{H}_{2}$, where Me is either Ti or $\mathrm{Zr}$. The term bistability means the co-existence of two stable travelling combustion waves.

The aim of the present paper is to investigate the behaviour of the competitive exothermic systems and to understand what is required for the existence of a multiplicity of flame fronts by which we mean flame fronts propagating (stable or otherwise) at different speeds for the same system parameters .

\section{MODEL \& MULTIPLICITY}

\subsection{Model}

We consider a diffusional-thermal model with two-step kinetics for premixed combustion wave propagation in one spatial dimension under adiabatic conditions. It is assumed that the reactant undergoes two competitive exothermic reactions: (R1) $A \rightarrow B$ and (R2) $A \rightarrow C$ with Arrhenius kinetics. The non-dimensional equations governing this process can be found in Towers et al. (2013) and are given as

$$
\begin{aligned}
& u_{t}=u_{x x}+v\left(e^{-1 / u}+q r e^{-f / u}\right), \\
& v_{t}=L^{-1} v_{x x}-v \beta\left(e^{-1 / u}+r e^{-f / u}\right),
\end{aligned}
$$

where $t$ and $x$ are non-dimensional time and space coordinates, $u$ and $v$ are the dimensionless temperature and fuel concentration, $\beta$ is the dimensionless activation energy of the first exothermic reaction (R1), $q$ is the ratio of the enthalpies, $r$ is the ratio of pre-exponential factors, $f$ is the ratio of the activation energies of the second (R2) to the first (R1) exothermic reaction respectively, and $L$ is the Lewis number for the fuel. For gaseous fuels $L=1$ and for solid fuels $L \rightarrow \infty$.

Equations (1) are considered subject to the boundary conditions

$$
\begin{array}{llll}
u=0, & v=1, & \text { for } & x \rightarrow \infty \\
u_{x}=0, & v_{x}=0, & \text { for } & x \rightarrow-\infty
\end{array}
$$

On the right boundary we have a cold $(u=0)$ and unburned state $(v=1)$. The non-dimensionalized ambient temperature is taken to be equal to zero. The assumption of zero ambient temperature is assumed here to 
circumvent the cold boundary problem, which has been discussed by many authors (e.g. see the discussion in Weber et al., 1997). On the left boundary $(x \rightarrow-\infty)$ neither the temperature of the mixture nor the concentration of fuel can be specified. We only require that there is no reaction occurring so the solution reaches a steady state of (1). Therefore the derivatives of $u$ and $v$ are set to zero for $x \rightarrow-\infty$.

We investigate travelling wave solutions to (1). Making the substitution $\xi=x-c t$, where $c$ is the constant speed of the travelling wave, the model (1) reduces to the system of ordinary differential equations

$$
\begin{aligned}
u_{\xi \xi}+c u_{\xi}+v\left(e^{-1 / u}+q r e^{-f / u}\right) & =0, \\
L^{-1} v_{\xi \xi}+c v_{\xi}-v \beta\left(e^{-1 / u}+r e^{-f / u}\right) & =0 .
\end{aligned}
$$

The system (3) consists of two second-order ordinary differential equation and in this sense is similar to the one-step models (Weber et al., 1997). However, in contrast to one-step models, here there is no additional integral, which can be used to reduce the order of the system (3) or to determine the downstream flame temperature as it is done in (Weber et al., 1997). Therefore the burning temperature, $u_{b}=\lim _{x \rightarrow-\infty} u(x, t)$ is undefined and should be considered as an eigenvalue of the problem as well as the flame speed.

The system of ordinary differential equations (3) together with boundary conditions (2) constitute a two-point boundary value problem, which is solved numerically by using a standard shooting algorithm with a fourthorder Runge-Kutta integration scheme first and then the results are corrected by employing the relaxation algorithm. Here the moving frame coordinate $\xi$ is discretized using finite differences. As the domain required by the travelling fronts varies greatly from one parameter set to the next $\xi$ is scaled to unity using an unknown parameter $\delta$. After providing an initial guess for the solution profile the resulting nonlinear algebraic equations are solved iteratively using the Newton-Kantorovich method until the change from one iteration to the next is less than $10^{-15}$. In addition to solving for $u$ and $v, \delta_{\xi}=0$ and $c_{\xi}=0$, for the scaling parameter $\delta$ and the front speed $c$ are added to the system and solved.

In practice, the shooting method is required only once to find a reasonable initial profile for the relaxation method. Once one solution for a particular parameter set was calculated, continuation methods were employed in conjunction with the relaxation method to find solutions for different parameters. When finding the curves presented in Figs. $2 \& 3$ the speed $c$ was the free parameter. By "scanning through" $c$, the activation energy $\beta$ (together with $u, v$ and other parameters) was calculated numerically and thus the curves are graphs of a function $\beta(c)$. The continuation methods work well in this case and there is no risk of missing the middle branch or failing to find a solution beyond one of the turning points.

\subsection{Cross-over temperature}

In this subsection we consider the conditions required for the existence of multiple solutions of system (3) for a given set of parameter values. Here, we will follow the work of Clavin et al. (1987) and introduce the cross-over temperature as a temperature, $u^{*}$, at which the rates of the two reactions are in balance. If the two terms for reaction rates in the second equation of system (3) are equated it is straightforward to show that $u^{*}=(f-1) / \ln r$. To make physical sense we require $u^{*}$ to be positive. If this is not the case and $u^{*}<0$ then one of the reactions is always dominating. By inspection we can see that in order to have a positive cross-over temperature we must have $\{f>1, r>1\}$ or $\{f<1, r<1\}$. These two parameter regions are really one as each can be mapped onto the other by a simple change of notation for the first and second reactions. In the remainder of this work we consider only $\{f>1, r>1\}$.

The first reaction dominates when the flame temperature is well below the cross-over temperature. In this situation the terms proportional to $r$ in system (3) can be neglected. This reduces the present model to the standard one-step model (Weber et al., 1997). For the one-step model flame speed and temperature can be derived by using asymptotic methods as

$$
u_{b}^{(1)}=\beta^{-1}, \quad c^{(1)}=e^{-\beta / 2} \sqrt{2 L \beta^{-1}}
$$

Alternatively, the second reaction dominates when the flame temperature is much higher than $u^{*}$. The first reaction terms in system (3) can be neglected and we once again derive the one step model, which after introducing new coordinate, $\xi^{\prime}=\xi \sqrt{r q / f}$, time, $t^{\prime}=t r q / f$ and activation energy, $\beta^{\prime}=\beta f / q$, can be written exactly in the same form as by Weber et al. (1997). After returning to the original variables, the asymptotic 


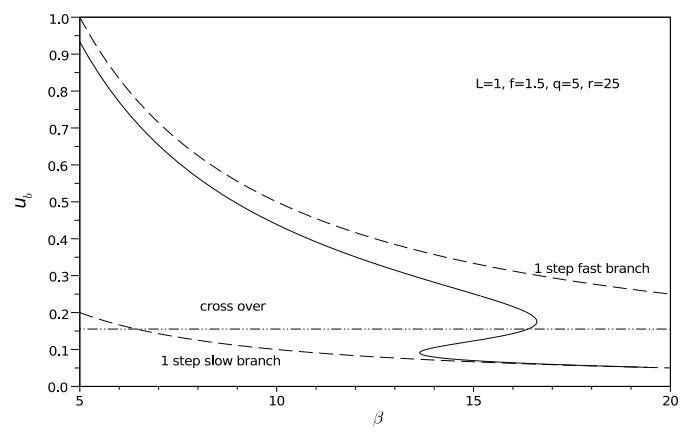

Figure 1. The burn temperature, $u_{b}$, against $\beta$ for $L=1, f=1.5, q=5$ and $r=25$. The two dashed lines indicate the variation of the burn temperature for one-step reactions equivalent to the fast and slow branches. The dot-dashed line labelled "cross over" indicates the cross-over temperature for this parameter set. Multiplicity of burning temperatures occurs near the cross-over temperature.

formulae (4) allow one to obtain the flame speed and temperature as

$$
u_{b}^{(2)}=q \beta^{-1}, \quad c^{(2)}=e^{-\beta f / 2 q} \sqrt{2 L r q^{2} \beta^{-1} f^{-2}} .
$$

By comparing the equations for the flame speed $(4,5)$ and definition of the cross-over temperature it is possible to derive two conditions for the activation energies,

$$
\beta^{(1)}=\ln r /(f-1), \quad \beta^{(2)}=q \ln r /(f-1),
$$

when the cross-over temperature is equal to the burning temperatures for the one-step models governed by reactions (R1) and (R2), respectively. Depending on the value of $q$ the temperatures, $u_{b}^{(1,2)}$, and activation energies, $\beta^{(1,2)}$, can be ordered in different ways.

When $q<1$ we must have $u_{b}^{(2)}<u_{b}^{(1)}$ and $\beta^{(2)}<\beta^{(1)}$. As we increase $\beta$ from the values where $u^{(2)} \gg u^{*}$ (or $\beta \ll \beta^{(1)}$ ) the reaction (R2) is dominating and the flame temperature follows the dependence in Eqn. (5). As $\beta$ approaches $\beta^{(2)}$ the reaction (R1) increases in significance. The flame temperature switches from the colder branch corresponding to (R2) to the hotter branch corresponding to (R1) as $\beta$ is increased from $\beta^{(2)}$ to $\beta^{(1)}$ in such a way that $u_{b}$ is staying close to $u^{*}$. For the values of the activation energy higher then $\beta^{(1)}$, the dependence $u_{b}(\beta)$ approaches the limiting behaviour (4) corresponding to (R1). Therefore when $q<1$ there is no multiplicity and the flame temperature is monotonic function of activation energy with a characteristic plateau behaviour in the region $\beta^{(2)}<\beta<\beta^{(1)}$ when neither reaction dominates .

If the situation is reversed and $q>1$, we have $u_{b}^{(2)}>u_{b}^{(1)}$ and $\beta^{(2)}>\beta^{(1)}$. This situation is depicted in Fig. 1 for $L=1, f=1.5, q=5$ and $r=25$. The dependencies $u_{b}^{(1,2)}(\beta)$ are plotted with the dashed lines, where index ' 1 ' corresponds to the curve marked as ' 1 step slow branch' and index ' 2 ' to the curve labelled ' 1 step fast branch' respectively. The dot-dashed line corresponds to the crossover temperature, $u^{*}$, which crosses the curve $u_{b}^{(1)}(\beta)$ at $\beta^{(1)} \approx 6.3$, while the intersection with $u_{b}^{(2)}$ is for $\beta^{(2)} \approx 32$ and is not shown in the figure. Numerical calculations of the temperature, $u$, for parameter values $L=1, f=1.5, q=5$, and $r=25$ are also plotted in Fig. 1 with the solid line. As the activation energy is increased from the values below both $\beta^{(1,2)}$ and the mixture is becoming less exothermic the flame temperature follows the formulae (5) for the one-step model governed by (R2). As the temperature decreases and approaches $u^{*}$ the first reaction "switches on" and the solution cannot follow the behaviour given by Eqns. (5). This causes the appearance of the first turning point close to the cross-over temperature. Further decrease of the burning temperature causes the solution to turn backward and to approach the slower and colder branch governed by reaction (R1), which results in folding and occurrence of the middle leg of the $u(\beta)$ dependence. As $u_{b}$ gets colder the second reaction freezes and the combustion wave is controlled by the reaction (R1). This causes the second turning point after which the solution rapidly approaches the limiting behaviour defined by Eqns. (4). This demonstrates that the condition $q>1$ is necessary for the existence of solution multiplicity. 


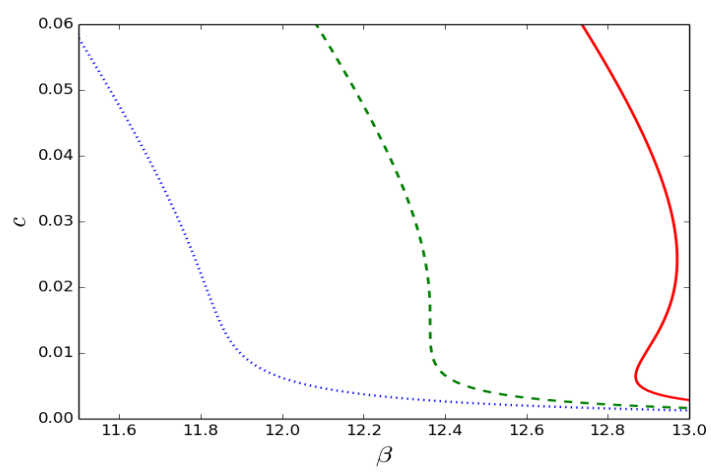

Figure 2. Three examples of flame front speed, $c$, plotted against activation energy, $\beta$ for $L=1, q=5$, $f=1.4$ and $r=5$ (dotted line) $r=5.69$ (dashed line) and $r=6.5$ (solid line). The curves demonstrate the transition from the existence of a unique solution for a given $\beta(r=5)$, through the transition point $r=5.69$, to multiplicity of solutions $r=6.5$ where the flame front speed exhibit the classic " $\mathrm{S}$ " curve.

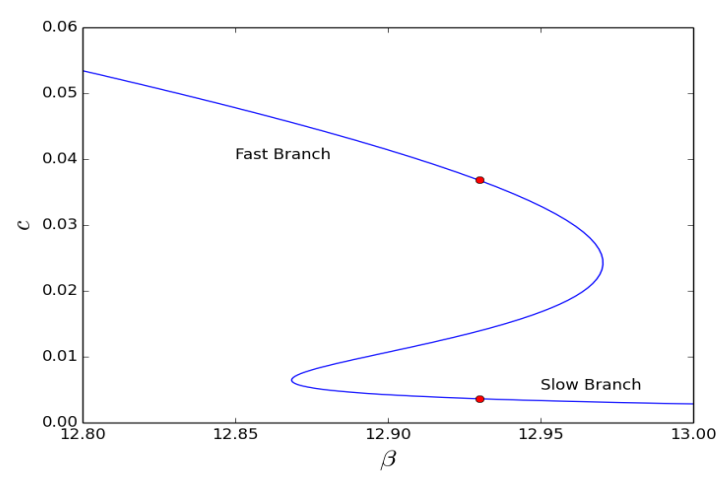

Figure 3. Flame front speed versus $\beta$ for $L=1, q=5, f=1.4$ and $r=6.5$. For most values of $\beta$ a single solution exists which is either part of the "fast" branch or "slow" branch of solutions. For a range of $\beta$ values, in this case $\beta \in(12.87,12.97)$, three solutions exist: one each from the fast and slow branches together with mid-branch solution which is aways unstable. The solid dots indicate the speeds of the travelling wave profiles in figure 4.

\section{RESULTS}

The travelling wave solutions are found numerically as detailed earlier. By solving system (3) with fixed $L, q, r$ and $f$ and using $c$ and $\beta$ and numerical continuation variables one can produce curves of flame front speed versus activation energy. Three such examples are in Fig. 2. For all three curves $L=1, q=5, f=1.4$ while $r \in(5,5.69,6.5)$. The dotted curve $(r=5)$ is a typical example of when a single solution exists i.e. there is only one speed a flame front can propagate at for a given activation energy $\beta$. The dashed curve $(r=5.69$ in Fig. 2 represents a threshold case as for larger values of $r$ a multiplicity of solutions exists. A typical example of a flame speed curve exhibiting multiplicity is shown by the solid line $(r=6.5)$. A close up of this curve is shown in Fig. 3. For $12.87<\beta<12.97$ there exists 3 different speeds for which a flame front may propagate. All solutions starting from the left of the figure up until the first turning point belong to the "fast" branch while the solutions after the second turning point constitute the "slow" branch. If the value of $r$ is further increased the ' $\mathrm{S}$ ' curve becomes more exaggerated and solution multiplicity exists for a larger range of $\beta$.

The solid dots in Fig. 3 indicate the solutions shown in Fig. 4. The flame front from the fast and slow branches are plotted against the scaled spatial dimension $\xi$ so they can be viewed on the same axes. The fast branch solution (dashed curve) is about 10 times faster than the slow branch solution (solid curve). The fast solution is also approximately $60 \%$ hotter in terms of the burn temperature. In terms of the unscaled spatial dimension $x$ 


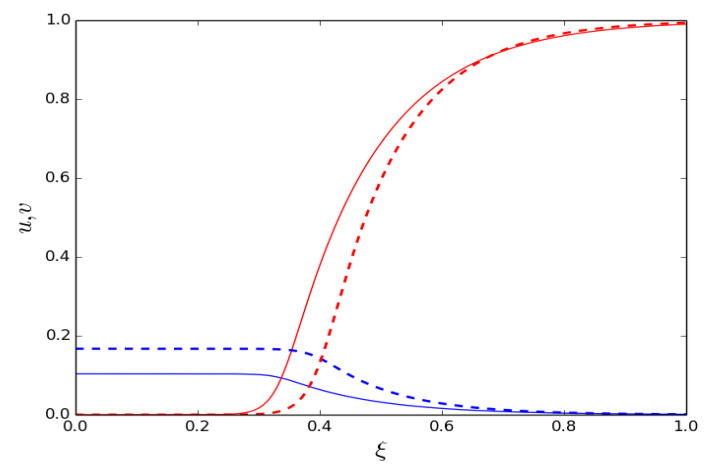

Figure 4. Temperature and fuel profiles from the fast branch (dashed lines) and slow branch (solid lines) for $L=1, q=5, f=1.4, r=6.5$ and $\beta=12.93$. The fast branch solution has a speed $c \approx 0.037$ while for the slow branch $c \approx 0.0036$. The spatial dimension of the profiles has been scaled to unity in order to directly compare both profiles. In the unscaled spatial dimension $x$ the slow branch profile is an order of magnitude wider than the fast branch profile.

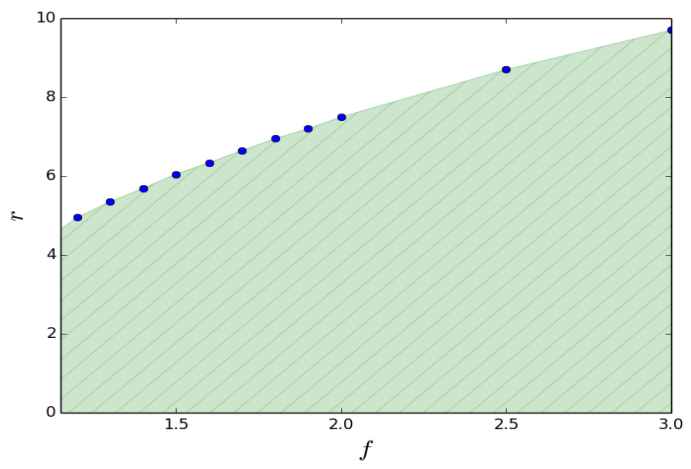

Figure 5. The region of unique solutions (shaded) and solution multiplicity (unshaded) in the $f-r$ plane for $L=1$ and $q=5$. The solid circles indicate the onset of multiplicity for specific values of $f$ and $r$.

the slow solution is an order of magnitude wider. Thus, although the parameters are the same the two solutions are quite different.

Using our numerical scheme we can find the values of $r$ and $\beta$ for the onset of solution multiplicity (dashed line in Fig. 2) for a fixed $L, q$ and $f$. By repeating the calculation for different values of $f$ we can discover the region in parameter space for multiplicity exists. Figure 5 shows this region for $L=1$ and $q=5$. The solid dots indicate the position in the $f$-r plane of the threshold for multiplicity at particular values of $f$. The shaded region indicates parameter values for which the flame front speed can only be single-valued. Crossing the boundary into the unshaded region in Fig. 5 the flame speed curve undergoes a folding into an ' $S$ ' similar to the example in Fig. 3. The deeper into the unshaded region of Fig. 5 one goes the more pronounced the ' $S$ ' becomes i.e. the larger the range of activation energies $\beta$ for which multiplicity exists. In the limit $f \rightarrow 1$ the boundary of the two regions tends to zero in Fig. 5. Only results for $L=1$ are presented but the position of the multiplicity threshold was found numerically to be only weakly dependent on $L$.

\section{CONCLUSIONS}

We have investigated a diffusional-thermal model describing a two-step competitive exothermic reaction scheme under adiabatic conditions in one spatial dimension. Such a model is relevant to the study of a practical physical process like self-propagating high-temperature synthesis (Markino, 2001). The governing PDEs were reduced to an ODE system using the travelling wave substitution and solved numerically using standard shoot- 
ing and relaxation schemes. Using the cross-over temperature concept necessary conditions for the existence of solution multiplicity were established. Numerical calculations have confirmed the existence of multiplicity and given the boundary of its onset. Increasing the ratio of the activation energies, $f$, has a significant effect on the value of $\beta$ for which multiplicity occurs. Increasing the ratio of pre-exponential factors, $r$, beyond the threshold value increases the range of activation energies which multiplicity exists. The issue of flame front stability has not been discussed in this paper and appears elsewhere (Towers et al., 2013).

\section{ACKNOWLEDGEMENT}

I.N.T acknowledges the hospitality and assistance of the P. N. Lebedev Physical Institute. V.V.G, A.V.K and A.A.P acknowledge the financial support from the Russian Foundation for Basic Research grants 11-01-00392 \& 13-03-00282. H.S.S acknowledges the support of the Australian Research Council Grant DP0878146.

\section{REFERENCES}

Ball, R., McIntosh, A. C. and Brindley, J. 1999. Thermokinetic models for simultaneous reactions: a comparative study, Combustion Theory and Modelling, 3, 447-468.

Berman V. S. \& Riazantsev Iu. S., 1975 Asymptotic analysis of stationary propagation of the front of parallel exothermic reaction. J. Appl. Math. Mech. 39 286-296. [In Russian: 1975 Prikl. Mat. Mekh. 39 306-315.]

Clavin P., Fife P. \& Nicolaenko I., 1987 Multiplicity and related phenomena in competing reaction flames. SIAM J. Appl. Math., 47, 296-331.

Gubernov V. V., Sharples J. J., Sidhu H. S., McIntosh A. C. \& Brindley J., 2012 Properties of combustion waves in the model with competitive exo- and endothermic reaction. J. Math. Chem. 50, 2130-2140.

Markino A., 2001 Fundamental aspects of the heterogeneous ame in the self-propagating high-temperature synthesis (SHS) process. Prog. Energy Combust. Sci. 27, 1-74.

Martirosyan N. A., Dolukhanyan S. K. \& Merzhanov A.G., 1983(a) Experimental observation of the nonuniqueness of stationary combustion in systems with parallel reactions. Combust. Explo. Shock, 19, 711-712. [In Russian: 1983 Fizika Goreniya i Vzryva 19 22-24.]

Martirosyan N. A., Dolukhanyan S. K., \& Merzhanov A.G. , 1983(b) Nonuniqueness of stationary states in combustion of mixtures of zirconium and soot powders in hydrogen. Combust. Explo. Shock 19,569-571. [In Russian: 1983 Fizika Goreniya i Vzryva 19 39-42.]

Please, C. P., Liu, F. and McElwain, D. L. S. 2003. Condensed phase combustion travelling waves with sequential exothermic or endothermic reactions. Combustion Theory and Modelling, 7, 129-143.

Sharples J. J, Gubernov V.V., Sidhu H.S , McIntosh A. C. \& Brindley J., 2012 Analysis of combustion waves arising in the presence of a competitive endothermic reaction, IMA J. Appl. Math., 77, 18-31.

Towers, I.N., Gubernov, V.V., Kolobov, A.V., Polezhaev, A.A. and Sidhu, H.S. 2013, Bistability of flame propagating in a model with competing exothermic reactions. Proc. R. Soc. Ser. A, 469, 20130315.

Weber R. O., Mercer G. N., Sidhu H. S. \& Gray B. F., 1997 Combustion waves for gases $\left(L_{e}=1\right)$ and solids $\left(L_{e} \rightarrow \infty\right)$. Proc. R. Soc. London Ser. A, 453, 1105-1118.

Westbrook, C.K. and Dryer, F. 1981. Simplified reaction mechanisms for the oxidation of Hydrocarbon Fuels in Flames, Combustion Science Technology, 27, 31-43.

Zeldovich Ya. B., Barenblatt G. I., Librovich V. B. \& Makhviladze G. M, 1985 The mathematical theory of combustion and explosion, Consultants Bureau, New York. 